\title{
Uso do assento de segurança infantil no banco traseiro de automóveis: análise em escolas de Fortaleza
}

\author{
Use of child restraint systems in car rear seats: \\ analysis in schools of Fortaleza
}

\author{
Alexandre Sá Pinto da Nóbrega Lucena ${ }^{1}$, Armando Nicodemos Lucena \\ Felinto ${ }^{1}$, Amanda Pires Bessa ${ }^{2}$, Artur Henrique Soares da Silva Filho ${ }^{3}$, \\ Crícia MLP Ferreira Gomes ${ }^{4}$, Yuri D da Rocha ${ }^{1}$, \\ Paulo Giordano Baima Colares ${ }^{1}$
}

DOI: http://dx.doi.org/10.11606/issn.2317-2770.v26i1p21-26

\begin{abstract}
Lucena ASPN, Felinto ANL, Bessa AP, Silva Filho AHS, Gomes CMLPF, Rocha YD, Colares PGB. Uso do assento de segurança infantil no banco traseiro de automóveis: análise em escolas de Fortaleza. Saúde, Ética \& Justiça. 2021;26(1):21-6.

RESUMO: Objetivo: Avaliar o uso do assento de segurança infantil, bem como o conhecimento da resolução do CONTRAN $\mathrm{n}^{\mathrm{O}}$ 277, de 2008, pelos pais e responsáveis de alunos de determinadas escolas na cidade de Fortaleza - CE. Métodos: Os autores estudaram de forma observacional e transversal os aspectos da utilização do assento de segurança infantil, servindose da aplicação de questionários acompanhados de termo de consentimento, esclarecendo aos participantes os motivos da pesquisa. Foram distribuídos questionários aos responsáveis pelos alunos de determinadas escolas de Fortaleza, dentre os quais um montante de 156 questionários foram devidamente preenchidos e utilizados na avaliação dos resultados da pesquisa. Resultados: De acordo com a análise dos 156 questionários respondidos no estudo, todos (100\%) os participantes sabem da existência de dispositivos de segurança para transporte de crianças em automóveis. Noventa e um por cento conhecem a resolução do CONTRAN n 277 de 2008, que regula o uso desses mecanismos. Dentre os participantes, 65,4\% sempre fazem uso dos assentos, ao passo que $28,9 \%$ os utilizam apenas algumas vezes e em certas ocasiões. Para $26,9 \%$ e $14,1 \%$ dos entrevistados, o preço e o tamanho, respectivamente, poderiam ser diminuídos para melhorar a adesão dos usuários aos dispositivos de retenção infantil. Conclusão: Dentre os participantes da pesquisa, $94,3 \%$ fazem uso constante ou ocasionalmente do assento de segurança infantil. O preço e o tamanho do dispositivo são os itens que mais desfavorecem o seu uso.
\end{abstract}

DESCRITORES: Criança; Sistemas de Proteção para Crianças; Acidentes de Trânsito.

\footnotetext{
1. Universidade de Fortaleza (Unifor), Fortaleza, CE

2. Hospital Sofia Feldman, Belo Horizonte, $\mathrm{MG}$

3. Hospital Municipal Odilon Behrens, Belo Horizonte, MG

4. Escola de Saúde Pública do Ceará, Fortaleza, CE

Endereço para correspondência: Armando Nicodemos Lucena Felinto. E-mail: armandonicodemos@hotmail.com
} 


\section{INTRODUÇÃO}

$\mathrm{A}$ cidentes envolvendo veículos automotores ocupam posição de destaque no perfil de mortalidade no Brasil e, dentre as causas externas, é a principal causa de morte no primeiro ano de $v_{\text {vida}}{ }^{1}$. Segundo dados do Sistema de Informações sobre Mortalidade - SIM, no ano de 2016, morreram em nosso país, vítimas de acidentes de transporte, 4.256 crianças e adolescentes na faixa etária de 0 a 19 anos$^{2}$.

A maioria dos traumatismos múltiplos envolvendo crianças em idade escolar são causados por acidentes automobilísticos, e mais da metade das crianças mortas nesses sinistros estava sem nenhuma forma de contenção no momento do trauma ${ }^{3}$.

Para minimizar as mortes e as possíveis sequelas de crianças passageiras de automóveis, foram desenvolvidos dispositivos de retenção infantil ou assentos de segurança infantil (ASI), com suas normas de utilização que, quando adequadamente aplicadas, reduzem em $71 \%$ a chance de morte ${ }^{4-6}$.

$\mathrm{O}$ uso dos ASI é regulamentado pela resolução do Conselho Nacional de Trânsito (CONTRAN) $\mathrm{n}^{\circ}$ 277 , de $2008^{7}$, tornando-o obrigatório para transporte veicular de crianças até 10 anos de idade. Entretanto, há dúvidas quanto à obediência da lei no país, observandose frequente negligência, não uso e uso errôneo do dispositivo ${ }^{8}$.

Este estudo consiste de uma análise do uso dos ASI no banco traseiro de automóveis, e tem como objetivo avaliar seu uso e o conhecimento da resolução do CONTRAN n ${ }^{\circ} 277$, de 2008, pelos pais e responsáveis de alunos de determinadas escolas na cidade de Fortaleza.

\section{MATERIAIS E MÉTODOS}

A análise qualitativa e quantitativa do uso de assentos infantis nos bancos traseiros de automóveis em Fortaleza foi feita em escolas particulares da cidade, levando em consideração a distribuição geográfica destas, de modo que houvesse abordagem de quatro das seis regionais da cidade.

Uma turma de cada nível escolar, com faixa etária entre 0 e 10 anos, foi escolhida aleatoriamente. O participante da pesquisa foi o responsável legal pelo aluno, e sua abordagem deu-se pela aplicação de um questionário composto por 28 questões, com o intuito de avaliar o uso do assento infantil nos bancos traseiros de automóveis, de acordo com a resolução do CONTRAN n ${ }^{\circ} 277$ de 2008, bem como identificar os possíveis problemas que estão impossibilitando o devido cumprimento legal dessa resolução.

Os questionários foram entregues aos responsáveis, juntamente com os termos de consentimento livre e esclarecido de participação da pesquisa, com o material do aluno. Foram respondidos e devolvidos sem identificação em envelopes lacrados disponibilizados pelos responsáveis pela pesquisa.

Foram devidamente respondidos um total de 156 questionários dentre todos os entregues. Toda a avaliação dos resultados foi focada nesse montante.

O estudo não trouxe riscos aos participantes, cujos anonimatos foram preservados. Os dados obtidos foram utilizados exclusivamente nesta pesquisa; e os resultados foram tornados públicos, fossem eles favoráveis ou não.

\section{RESULTADOS}

Ao término do estudo, foram analisados os 156 questionários devidamente preenchidos pelos responsáveis dos alunos, alcançando-se, pois, as conclusões a seguir.

Todos os 156 participantes do estudo declararamse cientes da existência de assentos específicos para o transporte de crianças em automóveis. Deste total, 142 (91\%) afirmaram ter conhecimento da resolução do CONTRAN n ${ }^{\circ} 277$ de 2008 (Tabela 1). Dez (6,4\%) participantes não conheciam tal resolução. Quatro pessoas $(2,6 \%)$ não opinaram neste quesito.

$\mathrm{O}$ conhecimento da referida resolução do CONTRAN, segundo dados da pesquisa, foi obtido através da televisão $(45,5 \%)$, da leitura de jornais $(16,7 \%)$, de amigos $(5,1 \%)$ e outros meios não mencionados $(3,2 \%)$.

Com relação à frequência do uso do assento nos automóveis, cento e duas $(65,4 \%)$ pessoas responderam que sempre utilizam o dispositivo. Vinte e quatro $(15,4 \%)$ entrevistados usam o assento apenas ocasionalmente. Catorze entrevistados $(9,0 \%)$ afirmaram que usam a cadeira de segurança infantil somente em viagens e sete pessoas $(4,5 \%)$ informaram usar o assento infantil somente em trajetos mais longos. Oito participantes $(5,1 \%)$ declararam nunca utilizar tal dispositivo (Tabela $2)$.

Tratando-se da motivação para o uso do assento infantil, oitenta $(51,3 \%)$ entrevistados afirmaram que utilizam o mecanismo em obediência ao código de trânsito, acreditando na necessidade deste. Cinquenta $(32,1 \%)$ entrevistados fazem uso pensando apenas na segurança da criança.

Ao analisar a abstenção do uso do mecanismo de segurança, questionando a frequência de vezes que o participante deixa de usar o assento, tem-se que quatorze entrevistados $(9,0 \%)$ raramente usam o dispositivo; vinte e sete $(17,3 \%)$ frequentemente o utilizam. Cento e duas $(65,4 \%)$ utilizam o assento sempre.

Dos entrevistados que não utilizam o dispositivo em seus veículos, sete $(4,5 \%)$ alegaram que este ocupa muito espaço no veículo. Seis $(3,8 \%)$ culparam a dificuldade para instalar e retirar o dispositivo do carro. Seis $(3,8 \%)$ acreditam que o dispositivo é estressante para 
Lucena ASPN et al. Uso do assento de segurança infantil no banco traseiro de automóveis: análise em escolas de Fortaleza.

a criança. Apenas duas pessoas (1,3\%) deixam de utilizar o assento levando em consideração o valor do material.

Quando os participantes do estudo foram inquiridos se alguém próximo do convívio já sofreu acidente lesionando a criança por não estar fazendo uso do dispositivo de retenção, foi apurado que quatorze (9\%) responderam: "sim (quanto à ocorrência de acidente com criança no autompovel), mas a criança não sofreu lesões, por estar usando a cadeira". Cento e dezesseis pessoas $(74,4 \%)$ responderam que nunca souberam de algum acidente desse tipo.

Ao serem indagados sobre o que poderia mudar nas cadeiras de segurança infantil para melhorar o uso, quarenta e dois $(26,9 \%)$ entrevistados marcaram o quesito "preço"; vinte e dois $(14,1 \%)$ assinalaram "tamanho"; dezenove $(12,2 \%)$ citaram a "forma de instalação"; seis $(3,8 \%)$ informaram que o material do dispositivo poderia ser melhorado para viabilizar o seu uso. Dezoito participantes $(11,5 \%)$ afirmaram que nada precisaria mudar para melhorar o uso do dispositivo - Gráfico 2.

TABELA 1 - Conhecimento da resolução do CONTRAN nº 277 de 2008

\begin{tabular}{lcc}
\hline & Número de participantes & Porcentagem \\
\hline Sim & 142 & 91,0 \\
Não & 10 & 6,4 \\
Não opinou & 4 & 2,6 \\
\hline Total & 156 & 100,0 \\
\hline
\end{tabular}

TABELA 2 - Freqüência de uso do assento infantil

\begin{tabular}{lcc}
\hline & Número de participantes & Porcentagem \\
\hline Sempre & 102 & 65,4 \\
Nunca & 8 & 5,1 \\
Às vezes & 24 & 15,4 \\
Somente para viagens & 14 & 9,0 \\
Somente em trajetos mais longos & 7 & 4,5 \\
Não opinou & 1 & 6,0 \\
\hline Total & 156 & 100,0 \\
\hline
\end{tabular}

GRÁFICO 1 - Meios de conhecimento da resolução do CONTRAN nº 277 de 2008

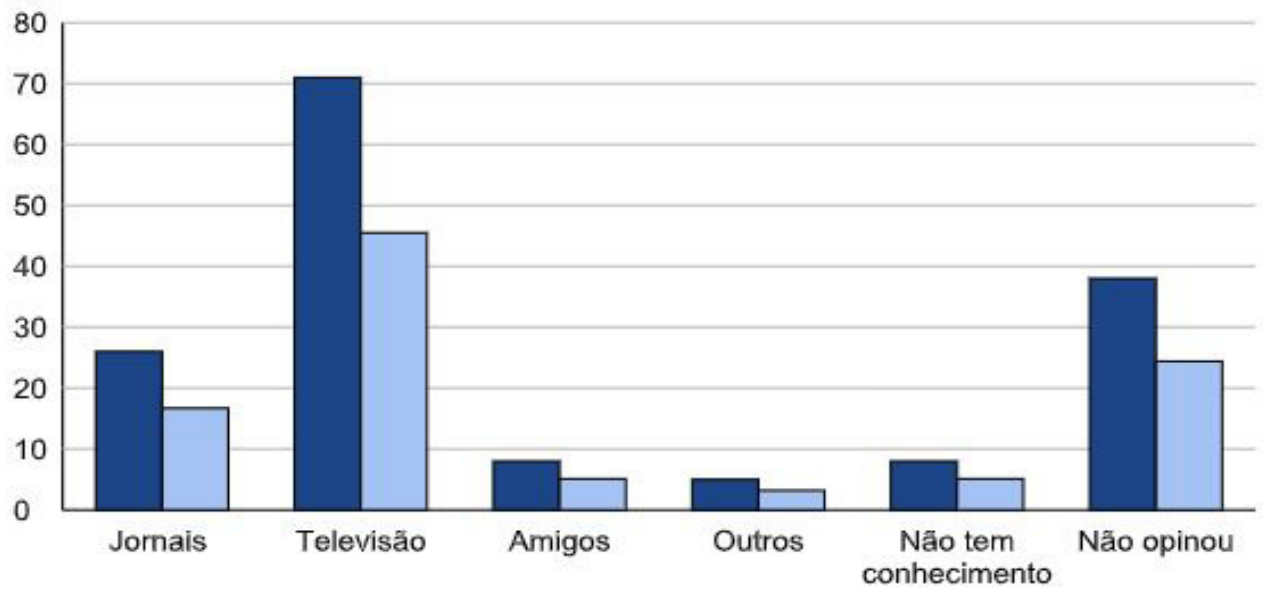


GRÁFICO 2 - Itens cuja mudança melhoraria a adesão ao assento infantil

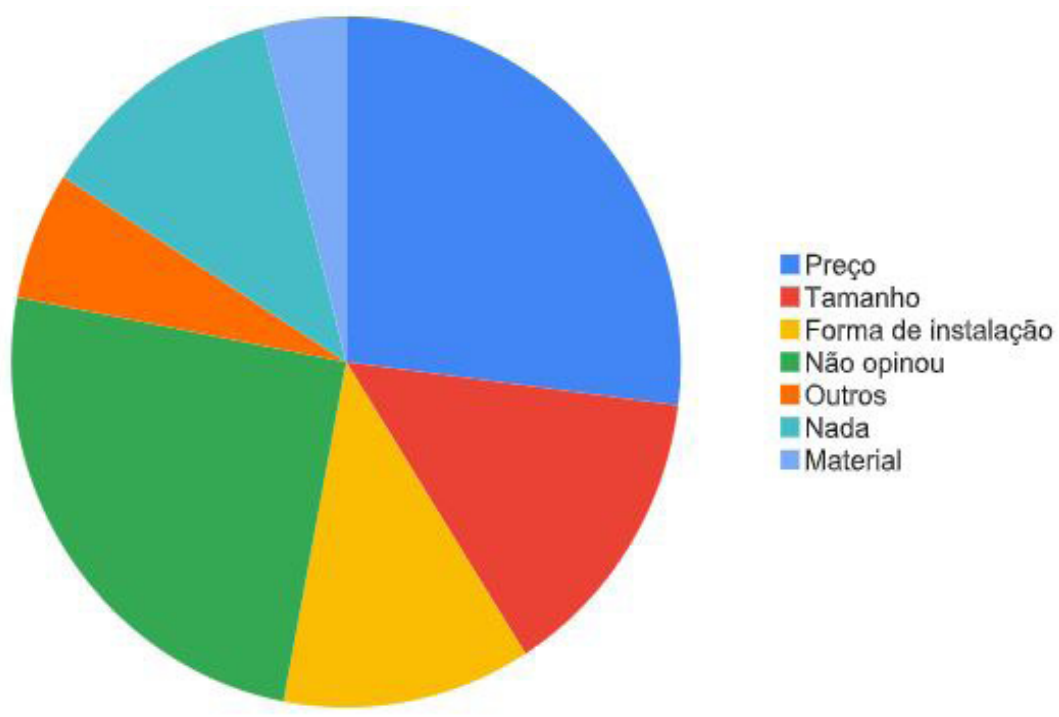

\section{DISCUSSÃO}

A resolução do CONTRAN n ${ }^{\circ} 277$ de 2008 dispõe sobre a utilização do dispositivo de retenção para o transporte de crianças em veículos, e a partir dela nos fundamentamos para análise dos critérios a serem seguidos para esse cuidado. Diante disso, a amostra se mostrou ciente em sua totalidade quanto à existência de assentos específicos para o transporte de crianças menores de 10 anos em automóveis. Contudo, quando perguntados sobre a obrigatoriedade do uso dos ASI, 91\% dos entrevistados demonstraram ter conhecimento da resolução do Conselho Nacional de Trânsito $n^{\circ} 277$, que regulamenta o uso do dispositivo e considera o não uso como infração gravíssima, passível de multa ${ }^{9}$.

Apesar do alto índice de conhecimento da legislação vigente e de sua obrigatoriedade, $65,4 \%$ da população estudada afirmou sempre usar o ASI durante o transporte, dado que mostra $34,6 \%$ de não adesão ou adesão parcial do dispositivo. Tal informação é compatível com as publicações globais e indica um elevado índice de negligência por parte dos responsáveis, além de uma discrepância entre conhecimento da legislação e sua prática $^{10,11,12,13,14}$.

Nesse estudo, o principal meio de veiculação de informações acerca dessa resolução foi a televisão $(45,5 \%)$, apontando uma fonte relevante para se trabalhar em projetos de divulgação e de esclarecimentos para melhor uso dos dispositivos de retenção ${ }^{15}$.

Quando questionados a respeito do motivo principal que os levou ao uso do ASI, 51,3\% dos entrevistados responderam que usavam o dispositivo porque estava de acordo com o Código de Trânsito
Brasileiro, acreditando ser uma mudança necessária; o que corrobora o fato de que as leis são um fator motivador para o uso de assentos de segurança ${ }^{16,17,18}$ e que, uma vez estando de acordo com a lei, os pais creem que a criança está sendo transportada de maneira apropriada ${ }^{19}$. Entretanto, 4,5\% dos inquiridos neste estudo afirmaram não utilizar o assento para realizar o transporte da criança.

Ao investigar os motivos que desencorajaram parte dos entrevistados a utilizar o dispositivo, foram apontados fatores inerentes a sua estrutura, como o grande tamanho, o incômodo que a proteção traz para a criança e a dificuldade para instalar e retirar o dispositivo. A posição do assento deve ser mais elevada em relação ao banco do carro, de forma que a altura da criança sentada se aproxime à altura de um adulto sentado, facilitando a contenção por cintos que envolvem bem a região dos ombros, tórax e cintura ${ }^{20}$, o que evita que a criança deslize sobre o banco, prevenindo lesões na cabeça, abdome e membros ("síndrome do cinto de segurança" 21,22,23).

No estudo, não foi relatado nenhum tipo de lesão provocada diretamente pelo uso do assento de segurança infantil. Durbin et al. ${ }^{24}$ também não encontraram lesões abdominais, torácicas ou de membros inferiores provocadas pelo assento, mas constataram que crianças que usavam apenas cinto de segurança apresentavam lesões em diversas áreas do corpo.

O custo dos dispositivos de retenção foi apontado como um dos fatores a ser melhorado. Cabe ao Governo financiar meios para baratear o custo destes assentos, pois, do ponto de vista societário, os benefícios claramente superam os custos ${ }^{25}$. 
Lucena ASPN et al. Uso do assento de segurança infantil no banco traseiro de automóveis: análise em escolas de Fortaleza.

\section{CONCLUSÃo}

O uso do assento de segurança infantil em Fortaleza mostrou-se aquém do esperado. Entender as causas de subaproveitamento desse dispositivo é essencial para o desenvolvimento de programas efetivos que orientem a população a respeito dos benefícios do seu uso, de forma a prevenir acidentes automobilísticos envolvendo crianças.

Lucena ASPN, Felinto ANL, Bessa AP, Silva Filho AHS, Gomes CMLPF, Rocha YD, Colares PGB. Use of child restraint systems in car rear seats: analysis in schools of Fortaleza. Saúde, Ética \& Justiça. 2021;26(1):21-6.

\begin{abstract}
Objective: To evaluate the use of child safety restraints, as well as to assess whether the parents and guardians of students in specific schools in Fortaleza - CE were aware of CONTRAN Resolution No. 277, of 2008. Methods: The authors studied observational and cross-sectional aspects of the use of child safety restraints, making use of questionnaires accompanied by a consent form explaining the reasons for participating in the research. A large number of questionnaires were distributed to students of specific schools in Fortaleza. A total of 156 questionnaires were fully completed and used in the analysis of the research results. Results: According to the analysis of 156 questionnaires completed in the study, all $(100 \%)$ participants were aware of the existence of safety devices for carrying children in cars. Ninety-one percent were familiar with the CONTRAN resolution No. 277, of 2008, which regulates the use of these mechanisms. Among the participants, $65.4 \%$ always made use of the seats, while $28.9 \%$ used them only sometimes and on specific occasions. To $26.9 \%$ and $14.1 \%$ of respondents the price and size, respectively, could be reduced to improve adherence to the use of child restraints. Conclusion: Among the survey participants, $94.3 \%$ made constant or occasional use of the child safety seat. The price and size of the device were the elements that most hindered its use.
\end{abstract}

KEY WORDS: Child; Child Restraint Systems; Accidents, Traffic.

\title{
REFERÊNCIAS
}

1. Barros MDA, Ximenes R, Lima MLC. Mortalidade por causas externas em crianças e adolescentes: tendências de 1979 a 1995. Rev Saúde Pública. 2001;35(2):142-9. DOI: https://doi.org/10.1590/S0034-89102001000200007

2. Carneiro FM. Estatísticas de acidentes de trânsito ocorridos entre 2016 e 2018 , com foco no número de mortes e faixa etária das vítimas [Internet]. Câmara dos Deputados; 2019. [Acesso em 2021 jun. 27]. Disponível em: estatisticas acidentes_Carneiro.pdf

3. Rockwood CA, Wilkins KE, Beaty JH, Kasser JR, editors. Rockwood and Wilkins' fractures in children. $6^{\text {th }}$ ed. Philadelphia: Lippincott Williams \& Wilkins; 2006.

4. Lund UJ. The effect of seating location on the injury of properly restrained children in child safety seats. Accid Anal Prev. 2005;37(3):435-9. DOI: https://doi.org/10.1016/j. aap.2004.12.004

5. Winston FK, Durbin DR, Kallan MJ, Moll EK. The danger of premature graduation to seat belts for young children. Pediatrics. 2000;105(6):1179-83. DOI: https://doi. org/10.1542/peds.105.6.1179

6. Arbogast KB, Durbin DR, Cornejo RA, Kallan MJ, Winston FK. An evaluation of the effectiveness of forward facing child restraint systems. Accid Anal Prev. 2004;36(4):5859. DOI: https://doi.org/10.1016/S0001-4575(03)00065-4

7. Brasil. Ministério da Infraestrutura. Conselho Nacional de Trânsito. Resolução CONTRAN no 854, de 08 de abril de 2021. Altera a Resolução CONTRAN no 816, de 17 de março de 2021, que dispõe sobre os prazos de processos e de procedimentos afetos aos órgãos e entidades do Sistema Nacional de Trânsito e às entidades públicas e privadas prestadoras de serviços relacionados ao trânsito, por força das medidas de enfrentamento da pandemia de Covid-19 no Estado do Acre [Internet]. Brasília, DF; 2021. [Acesso em 2021 mai. 27]. Disponível em: https://www.in.gov. br/en/web/dou/-/resolucao-contran-n-854-de-8-de-abrilde-2021-313199394\%20

8. Oliveira SRL, Carvalho MDB, Santana RG, Kawazoe $\mathrm{CH}$, Santos EQ, Oliveira FC. Análise de metodologias de pesquisa sobre utilização de assentos de segurança infantil: lições de um projeto piloto. Rev paul pediatr. 2008;26(2):119-23. DOI: https://doi.org/10.1590/S010305822008000200004

9. Brasil. Presidência da República, Subchefia para Assuntos Jurídicos. Lei $n^{\circ}$ 9.503, de 23 de setembro de 1997. Institui o Código de Trânsito Brasileiro [Internet]. Brasília, DF; 1997. [Acesso em 2021 mai. 27]. Disponível em: http:// www.planalto.gov.br/ccivil_03/Leis/L9503Compilado.htm

10. Yanchar NL, Kirkland SA, LeBlanc JC, Langille DB. Discrepancies between knowledge and practice of childhood motor vehicle occupant safety in Nova Scotia: A population-based study. Accid Anal Prev. 2012;45:326-33. DOI: https://doi.org/10.1016/j.aap.2011.07.020

11. Scheidler MG, Shultz BL, Schall L, Ford HR. Risk factors and predictors of mortality in children after ejection from motor vehicle crashes. J Trauma. 2000;49(5):864-8. DOI: https://doi.org/10.1097/00005373-200011000-00012

12. Greenberg-Seth J, Hemenway D, Gallagher SS, Ross JB, 
Lucena ASPN et al. Uso do assento de segurança infantil no banco traseiro de automóveis: análise em escolas de Fortaleza.

Lissy KS. Evaluation of a community-based intervention to promote rear seating for children. Am J Public Health. 2004;94(6):1009-13. DOI: https://doi.org/10.2105/ ajph.94.6.1009

13. Winston FK, Chen IG, Smith R, Elliott MR. Parent driver characteristics associated with sub-optimal restraint of child passengers. Traffic Inj Prev. 2006;7(4):373-80. DOI: https://doi.org/10.1080/15389580600789143

14. Pickrell TM, Ye T. Child restraint use in 2008: use of correct restraint types. Washington, DC: National Center for Statistics and Analysis, National Highway Traffic Safety Administration; 2009.

15. Olufunlayo TF, Odeyemi KA, Ogunnowo BE, Onajole AT, Oyediran MA. An observational survey of child car safety practices in private pre-primary and primary schools in two local government areas of Lagos State, Nigeria. Inj Prev. 2012;18(4):216-20. DOI: https://doi.org/10.1136/ injuryprev-2011-040047

16. Ebel BE, Koepsell TD, Bennett EE, Rivara FP. Too small for a seatbelt: predictors of booster seat use by child passengers. Pediatrics. 2003;111(4 Pt. 1):e323-7. DOI: https://doi.org/10.1542/peds.111.4.e323

17. Bingham CR, Eby DW, Hockanson HM, Greenspan AI. Factors influencing the use of booster seats: A state-wide survey of parents. Accid Anal Prev. 2006;38(5):1028-37. DOI: https://doi.org/10.1016/j.aap.2006.04.014

18. Simpson EM, Moll EK, Kassam-Adams N, Miller GJ, Winston FK. Barriers to booster seat use and strategies to increase their use. Pediatrics. 2002;110(4):729-36. DOI: https://doi.org/10.1542/peds.110.4.729

19. Child passenger safety survey commissioned by DaimlerChrysler with the support of NHTSA. Alexandria, VA: Public Opinion Strategies; 2000.

20. Arbogast KB, Jermakian JS, Kallan MJ, Durbin DR. Effectiveness of belt positioning booster seats: an updated assessment. Pediatrics. 2009;124(5):1281-6. DOI: https:// doi.org/10.1542/peds.2009-0908

21. Kulowski J, Rost WB. Intra-abdominal injury from safety belt in auto accident: report of a case. AMA Arch Surg. 1956;73(6):970-1. DOI: https://doi.org/10.1001/ archsurg.1956.01280060070015

22. Garret JW, Braunstein PW. The seat belt syndrome. J Trauma. 1962;2:220-38. DOI: https://doi.org/10.1097/00005373196205000-00002

23. Agran PF, Dunkle DE, Winn DG. Injuries to a sample of seatbelted children evaluated and treated in a hospital emergency room. J Trauma. 1987;27(1):58-64. DOI: https://doi.org/10.1097/00005373-198701000-00011

24. Bhatia E, Holmes JH, Shaw KN, Werner JV, Sorenson W, Winston FK. Partners for child passenger safety: a unique child-specific crash surveillance system. Accid Anal Prev. 2001;33(3):407-12. DOI: https://doi.org/10.1016/s00014575(00)00054-3

25. Miller TR, Zaloshnja E, Hendrie D. Cost-outcome analysis of booster seats for auto occupants aged 4 to 7 years. Pediatrics. 2006;118(5):1994-8. DOI: https://doi. org/10.1542/peds.2006-1328 